\title{
COVID-19: Look to the Future, Learn from the Past
}

\author{
Zhangkai J. Cheng ${ }^{1,2,3}$, Zhigang Liu' ${ }^{2}$, Ruixi Zeng ${ }^{4}$, Lifeng Tian ${ }^{1}$, Zhifeng Duan ${ }^{1}$, Hakon Hakonarson ${ }^{1,5}$, \\ Hui-Qi Qu ${ }^{1}$, Qing Zhang ${ }^{6}$, Liteng Yang ${ }^{7}$, Gang Cheng ${ }^{4, *}$
}

1. Center for Applied Genomics, The Children's Hospital of Philadelphia, Philadelphia, USA

2. Institute of Allergy and Immunology, School of Medicine, Shenzhen University, Shenzhen, China

3. Institute of Medical Physics, University of Sydney, Sydney, Australia

4. Department of Plastic Surgery, The First Affiliated Hospital, Sun Yat-sen University, Guangzhou, China

5. Department of Pediatrics, University of Pennsylvania Perelman School of Medicine, Philadelphia, USA

6. Tissue and Organ Bank, General Hospital of Ningxia Medical University, Yinchuan, China

7. Respiratory Department, The Third Affiliated Hospital, Shenzhen University, Shenzhen, China

*drchenggang@tom.com

Keywords: coronavirus; epidemiology; 2019-nCoV; COVID-19;

\begin{abstract}
Background: There is a current worldwide outbreak of a new type of coronavirus COVID-19. The number of confirmed infected cases is rapidly increasing.

Method: This paper analyzes the characteristics of COVID-19 in comparison with Severe Acute Respiratory Syndrome Coronavirus (SARS-CoV), Middle East Respiratory Syndrome Coronavirus (MERS-CoV) and influenza. Diagnostic data for foreign citizens evacuated from Wuhan were collected and compiled. Current prevention and control strategies have been analyzed.
\end{abstract}

Results: COVID-19 is similar to SARS-CoV and MERS-CoV virologically and etiologically, but similar to influenza in epidemiology and virulence. The prevalence rate in Wuhan was inferred to be close to $1 \%$. The comparison provides a new perspective for the future of the disease, and offers some advice in the prevention and control management strategy.

Conclusion: The large number of patients and the strong occult nature are two big problems, making the virus difficult to eradicate. We need to contemplate the possibility of long-term co-existence with COVID-19.

\section{Introduction}

In December 2019, a cluster of patients presenting with viral pneumonia were reported in Wuhan, China. This was identified to be caused by a new type of coronavirus, COVID-19. The coronavirus quickly spread across people living in or visited Wuhan, and human-to-human transmission was soon confirmed. For a more detailed description of the outbreak, please refer to our previous study on the virology, epidemiology and clinical guidelines (1).

The virus is spreading rapidly. As of $13^{\text {th }}$ February 2020 , the confirmed number of cases in China as reported by the Chinese National Health Commission has risen up to 52526 , with $15 \%$ being severe cases, and 1367 fatalities (2). Furthermore, the coronavirus has spread overseas to 24 other countries (3). Figure 1 traces the development of the infection over time, with data taken from the past daily statements of National Health Commission (2). The sudden spike in the confirmed infection number seen on the $13^{\text {th }}$ February is due to Hubei province including the number of clinical cases (but not confirmed by polymerase chain reaction, or PCR test) into the number of confirmed cases published. 
We study the characteristics of COVID-19 in comparison with Severe Acute Respiratory Syndrome Coronavirus (SARS-CoV), Middle East Respiratory Syndrome Coronavirus (MERS-CoV) and influenza, in terms of virology, etiology, epidemiology and virulence. Prevention and control strategies are analyzed and discussed.

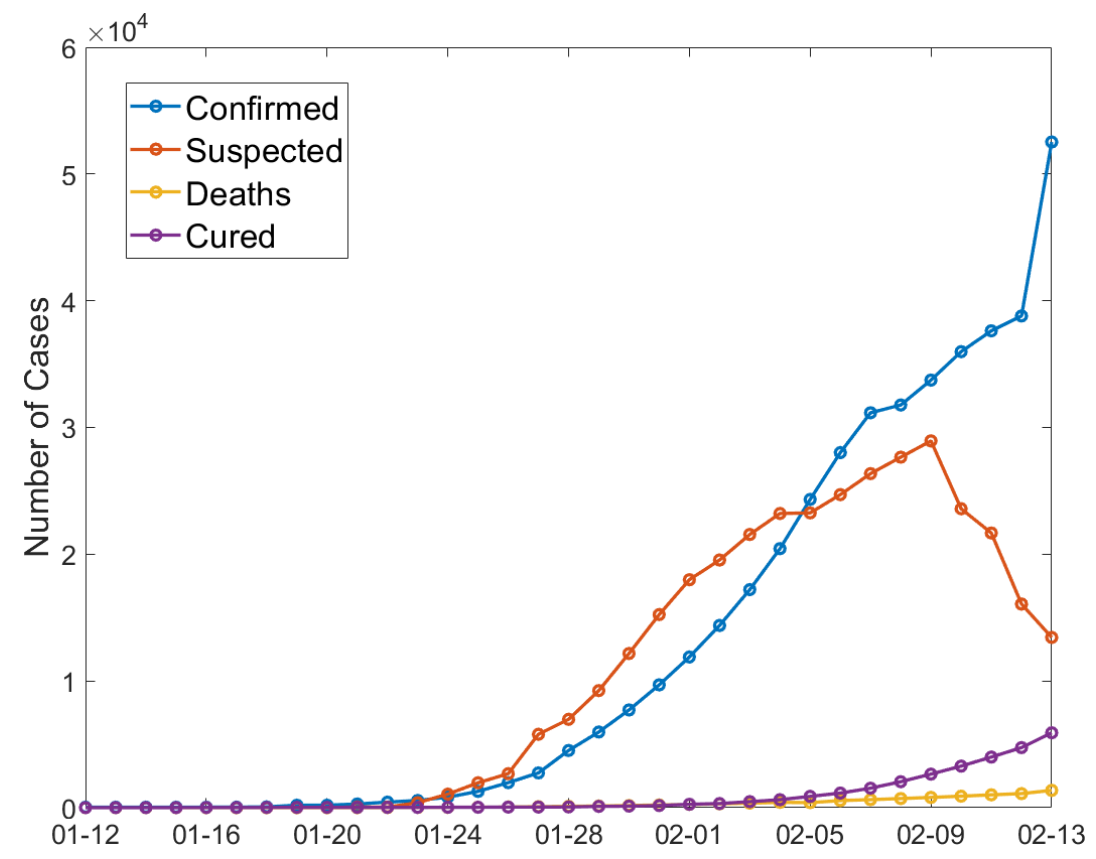

Figure 1. Number of confirmed cases, suspected cases, deaths and cure in China over time.

\section{Comparison to SARS-CoV and MERS-CoV}

After Severe Acute Respiratory Syndrome Coronavirus (SARS-CoV) and Middle East Respiratory Syndrome Coronavirus (MERS-CoV), COVID-19 is the seventh known member of the family of coronaviruses that are pathogenic to humans. The rest of the seven members are associated with mild clinical symptoms (4). SARS-CoV emerged in China in November 2002, and resulted in more than 8000 human infections and 774 deaths in 37 countries $(5,6)$. MERS-CoV was first detected in Saudi Arabia in 2012 and was responsible for 2494 confirmed cases of infection and 858 fatalities, including 38 deaths following a single introduction into South Korea (7-9). COVID-19 (GenBank accession $\mathrm{MN908947)}$ is a betacoronavirus ( $\beta \mathrm{CoV}$ ) of group $2 \mathrm{~B}$, and was found to have the highest similarity (89\%) to a SARS-related member of the Sarbecoviruses (GenBank accession MG772933), a subgenus within the $\beta$ CoV genus $(10,11)$.

Early patient data from Wuhan in China has noted similarities of clinical features between COVID-19 and previous $\beta \mathrm{CoV}$ infections. Similar to SARS-CoV and MERS-CoV infections, patients exhibited symptoms of viral pneumonia including fever, cough, difficulty breathing and bilateral ground-glass opacities on chest CT scans (12). However, the virulence of COVID-19 is far less than that of SARS, with the current running fatality rate around $2 \%$, and even this figure should have been overestimated due to the large number of unconfirm cases, whereas the case fatality rate is around $10 \%$ for SARS (13) and $37 \%$ for MERS (14).

Similar to the 2003 SARS outbreak in Guangzhou in China, both outbreaks were initially connected to "wet markets" where wildlife animals and meat were sold $(1,15)$. During previous outbreaks of SARS 
and MERS, human to human transmission was efficient through droplets, contact and fomites, suggesting that the transmission mode of the COVID-19 can be similar (16).

In terms of transmissibility, we previously studied the reproduction number Ro provided by eight different research groups, and found most of the $R_{0}$ value to lie in the range between 2 and 3 (1). On $31^{\text {st }}$ January 2020, scientists at the University of Hong Kong published in The Lancet the most comprehensive model yet for predicting the future spread of COVID-19 and estimated $\mathrm{R}_{0}$ to be around 2.68 (17). This $R_{0}$ estimation is similar to or slightly less than that of SARS (around 3) (18) and larger than MERS, which is less than one (19).

COVID-19 is similar in transmissibility compared to SARS, while being much less virulent than SARS. Why, then, has the number of confirmed cases of the disease greatly surpassed SARS and continued to increase? This has been for a variety of epidemiological reasons:

1. Detection, description, and warning measures lagged far behind in the early stages of the outbreak, which gave time for the virus to spread. As a result, before the large-scale national prevention and control measures were implemented, especially before the closure of Wuhan, a large number of patients had been accumulated in the Wuhan area. Due to technical factors such as medical resource overload and shortage of diagnostic materials, the current statistics of Hubei and Wuhan are lagging behind, thus the number of patients in Wuhan and across the country may be greatly underestimated. The Lancet paper of January 31 estimates that as of January 25, there were more than 75000 patients in Wuhan alone (17). After Wuhan was closed down, a few governments evacuated their nationals and performed thorough medical examination for each citizen brought back. The authors searched available information on Wuhan evacuation released by governments on the public domain. Table 1 lists the citizens evacuated by each country in the past two weeks. Evacuation can be thought of as a random sampling of the population of the Wuhan area (11 million in total), and the prevalence rate is close to what the Lancet paper suggested. In other words, the infection rate in the Wuhan area may have reached one percent, or 110000 people.

\begin{tabular}{|c|c|c|c|c|c|}
\hline Country & Evacuated & $\begin{array}{c}\text { Confirmed } \\
\text { Cases }\end{array}$ & Prevalence & $\begin{array}{c}\text { Converted to infected } \\
\text { population in Wuhan }\end{array}$ & Source \\
\hline Japan & 566 & 9 & $1.4 \%$ & 156000 & $(20)$ \\
\hline South Korea & 368 & 1 & $1.4 \%$ & 150000 & $(21-23)$ \\
\hline US & 195 & 0 & 0 & 0 & $(24)$ \\
\hline Germany & 124 & 2 & $1.6 \%$ & 177000 & $(25)$ \\
\hline Singapore & 92 & 1 & $1.1 \%$ & 119000 & $(26-28)$ \\
\hline Italy & 56 & 1 & $1.8 \%$ & 196000 & $(29)$ \\
\hline Total & 1401 & 14 & $1.0 \%$ & 110000 & NA \\
\hline
\end{tabular}

Table 1. Citizens evacuated from Wuhan by each country.

2. Timing. Both the COVID-19 outbreak in Wuhan and 2003 SARS outbreak in Guangzhou happened in major cities in China. Around January each year, a huge amount of population would leave major cities and travel back to their respective hometown, and come back around February. This is known as the Chunyun period, or Spring Festival travel season. The COVID-19 outbreak happened in late December and early January, albeit the low number of confirmed cases due to lack of detection. Before Wuhan closed its city, 5 million people already left Wuhan during the Chunyun period (30), which hinders any containment effort. SARS on the other hand, although detected in 2002, the real outbreak did not happen until March 2003. Chunyun period had by then ended, and the containment efforts were effective. 
3. The occult nature of COVID-19. COVID-19 is not as virulent as SARS, and can produce a large number of patients with mild symptoms, who are likely not to go to hospitals for treatment, and even if they do, they may not be formally diagnosed, leading to a low number of confirmed cases. A recent report in Caijing Magazine shows the existence of a large number of unreported cases (31). At the same time, the incubation period of new coronavirus pneumonia is longer than SARS, generally between 2-10 days (32). Furthermore, a risk of pre-symptomatic transmission was suggested by Rothe and colleagues (33), however, this finding was met with criticisms (34). SARS on the other hand, exhibits serious illness, resulting in a lower chance of mis-reporting.

\section{Etiology}

Most of the first cluster of patients reported by the Wuhan Municipal Health Commission (WMHC) in China all had a history of exposure in the Huanan Seafood Wholesale Market, suggesting that the virus originated from wildlife animals sold in the market (35). However, a later Lancet report found 13 of the 41 first reported patients with no epidemiological link to the marketplace (12). The virus may have spread silently in Wuhan before the discovery of the cases, during its incubation period $(36,37)$.

All three aforementioned viruses (SARS-CoV, MERS-CoV and COVID-19) are believed to have natural hosts in bats $(38,39)$. But most likely, they were able to invade the human world only after a genetic mutation in an intermediate host animal. The intermediate hosts for SARS and MERS are civets (40) and camels (41), respectively. Determining the intermediate host is crucial to our future efforts to prevent the return of COVID-19. Unfortunately, scientists were only able to detect the virus in the market environment, but not in time to collect wildlife samples before the market was closed (35). There has been various candidates proposed, but most of them have been discredited (42). On $7^{\text {th }}$ February 2020, South China Agricultural University held a press release and announced that they have found a potential candidate for intermediate host and provided solid evidence for it. By analyzing more than 1,000 metagenomic samples, the research team identified pangolins as potential intermediate hosts for COVID-19. The positive rate of COVID-19 in pangolin was $70 \%$ by molecular biological tests. The virus was further isolated and identified, and the typical coronavirus particle structure was observed under electron microscope. Finally, through the analysis of the virus genome, it was found that the sequence similarity between the isolated virus strain and the current infected human strain was up to $99 \%$ (43). This finding is of great significance for the prevention and control of the outbreak at the source, and provide scientific basis for the adjustment of relevant policies on wildlife control.

\section{Prevention and Control}

Learning from the SARS outbreak, which started as animal-to-human transmission during the first phase of the epidemic, all game meat trades should be optimally regulated to terminate this portal of transmission. Regulation and cracking down on wildlife trafficking should be an important disease control measure (44).

Global experts and governments are actively tracking the spread and virulence of the virus, and to provide advice to individuals on measures to protect health and prevent the spread of this outbreak. In response to the spreading virus, isolation strategy has been used. Many Chinese cities including Wuhan have been put on quarantine since $23^{\text {rd }}$ January, 2020, many countries have closed their boarder to people travelled from China (1). 
To systematically organize countries to combat COVID-19, WHO has developed a "global strategic preparedness and response plan" to support all countries to prepare for and respond to the outbreak. The goal is to stop further transmission of COVID-19 within China and to other countries, and to mitigate the impact of the outbreak in all countries. They have outlined a list of public health measures, including rapid identification, diagnosis and management of the cases, identification and follow up of the contacts, infection prevention and control in healthcare settings, implementation of health measures for travelers, awareness raising in the population, risk communication (16).

The understanding of the virus is still updating rapidly, but that does not prevent us from starting to think further about the problem of the future and preparing plans for it.

\subsection{A New Perspective}

Effectively and comprehensively isolating patients and their close contacts, cutting off their path to continuing transmission, and waiting for the incubation period to pass, was a very effective measure to eliminate SARS in a short period of time. This strategy may work on several premises:

1. The total number of patients was small. Therefore, we were able to find and isolate the close contacts of each patient;

2. Once becomes onset, the condition was critical and typical. Patients were quickly identified and treated;

3. No transmission ability during incubation period.

COVID-19 however, may not satisfy these preconditions. Having a huge number of patients as well as strong occult greatly increased the difficulty of prevention and control. We will not be able to put every patient that exhibits mild symptoms into quarantine, and to put all their close contacts under isolation and observation.

On another hand, COVID-19 shares many similar characteristics of influenza. Here are the features of the seasonal flu, which kills 646,000 people worldwide each year (45): It has low virulence, with case fatality rate of about $0.1 \%$ (46); Its reproduction number $\mathrm{R} 0$ is around 1.3, which is less than SARS (47); The number of cases is much larger. About $10 \%$ of unvaccinated adults are infected each year; Although influenza virus usually has a short incubation period of between 1 - 4 days, transmission can occur within the incubation period (48); Flu symptoms are less distinct, with fever, cough, sore throat and other symptoms that are difficult to distinguish from other respiratory diseases prevalent in autumn and winter. Table 2 below compares different characteristics of the three coronaviruses, as well as influenza.

\begin{tabular}{|c|c|c|c|c|}
\hline & COVID-19 & SARS-CoV & MERS-CoV & Influenza \\
\hline Ro & 2.68 & 3 & $<1$ & 1.3 \\
\hline Virulence & Low & High & High & Low \\
\hline Case Fatality Rate & $2 \%$ & $10 \%$ & $37 \%$ & $0.1 \%$ \\
\hline Natural Hosts & Bat & Bat & Bat & Animals, humans \\
\hline Intermediate Hosts & Pangolins & Civets & Camels & NA \\
\hline Origin of Outbreak & Wuhan, China & Guangzhou, China & Saudi Arabia & NA \\
\hline Incubation Period & $2-10$ days & $2-7$ days & $4-8$ days & $1-4$ days \\
\hline
\end{tabular}

Table 2. Comparison of four diseases. 
Although COVID-19 is similar to SARS in virology and etiology, its epidemiological impacts are less like SARS and more like a "mega flu". This means we need to take historical experiences from the fight against influenza.

Closing schools during flu outbreaks has been shown to be an effective measure $(49,50)$. However, when to shut down and how long to shut down are still indicators that need to be carefully considered (51). Two studies from Australia and Japan showed that monitoring body temperature at airports was not effective in reducing the spread of the disease during the $2009 \mathrm{H} 1 \mathrm{~N} 1$ swine flu pandemic $(52,53)$. A similar question to consider is how much effort we need to put into monitoring passengers' temperatures in this outbreak, given that the incubation period for COVID-19 is much longer than for influenza.

\subsection{Vaccines}

Vaccines are the first choice against a pandemic of this magnitude. According to U.S Centers for Disease Control and Prevention (CDC), the effectiveness of the flu vaccine varies from year to year, but overall it reduces the risk by 40 to 50 percent (54). Vaccines need to go through many phases of development. The lead time to produce a vaccine lot ranges from several months (e.g. influenza vaccine) to three years (55). Thanks to the rapid publication of the COVID-19 genetic sequence, a number of biopharmaceutical firms and academic research facilities around the world have launched programs and are racing to find an effective vaccine. Many are aiming to break the vaccine development record time (56).

\subsection{Hierarchical Management}

"Hierarchical management" takes into account the fact that when influenza is endemic, outbreaks in different parts of the world vary from region to region, and the severity of illness varies from patient to patient. Cities with clusters of cases may take more drastic measures, such as canceling public gatherings and even closing schools and workplaces, while areas with fewer cases can adapt to local conditions. Patients with serious illness are given intensive treatment in hospitals, while most patients with mild symptoms can seek treatment in small clinics or even wait for the disease to self-heal.

This idea can certainly be translated to fight against COVID-19. During a full-scale pandemic, as seen in the city of Wuhan, there will not be enough medical resources to attend every patient (57). Medical needs should be given out to those with more severe symptoms. To treat the large number of patients with mild symptoms, the solution is to create makeshift hospitals using empty facilities such as sports stadium and exhibition halls, which is what Wuhan is doing (58). Patients with less severe symptoms are gathered and assigned a bed and facemasks in the makeshift hospital, and less medical resource is needed for each patient.

The current distribution of the disease is centered in Wuhan, Hubei (see Figure 2) and its surrounding cities, while cases in other cities are mainly imported. It may take a long time and very strict prevention and control measures until the outbreak in Wuhan is effectively controlled. In other areas however, the situation is expected to be largely controlled by next week, due to the isolation strategy in China after $23^{\text {rd }}$ January 2020. Within 14 days after the closure of Wuhan in Hubei Province, potential patients in other cities would have already developed clinical symptoms, with high probability of being quarantined and treated. At that point, it may be more important to consider putting the society back 
into normal operation and function as soon as possible. We should exercise specific disease control and management in different regions, instead of a "one-size-fits-all" approach.

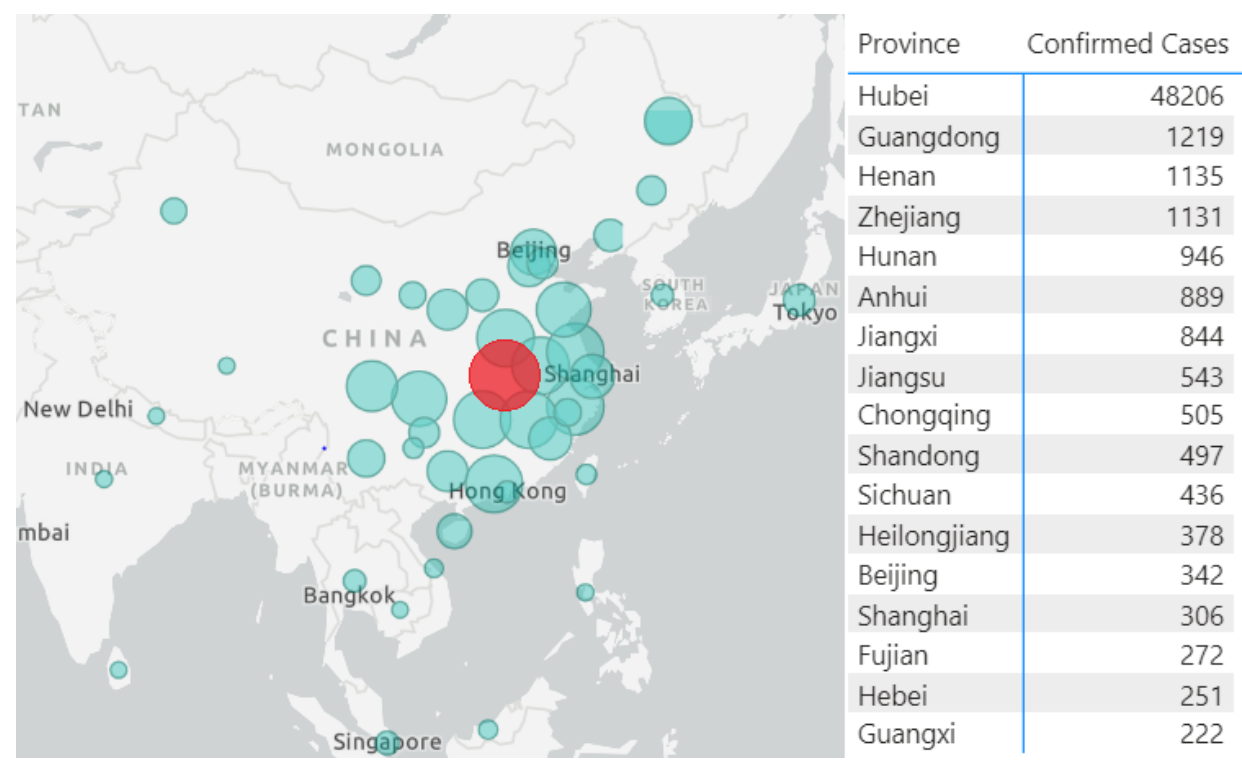

Figure 2. Bubble map visualization of the disease distribution around China, with data taken from (3), obtained on $13^{\text {th }}$ February 2020. Created using Power BI Desktop.

\subsection{A long winter}

Governments around the world are doing their best in combatting COVID-19, and it is probable that we can eradicate the virus comes summer, as with the fight against SARS. After the temperature warms up in summer, it will be harder for the virus to survive in the summer heat. However, we still need to be prepared for a long epidemic. The development of COVID-19 is closer to a "mega flu" that is more difficult to eradicate. That brings a possibility that this virus, like the flu, will persist in the human world for a long time to come. Humans are still at a loss for influenza viruses that are less transmissible than COVID-19, and given the early accumulation of cases in the Wuhan area, it may be necessary to start embracing the possibility of long-term co-existence with COVID-19.

That possibility may not be as scary as it sounds. The case fatality rate for COVID-19 has fallen from an initial 15 percent to about 2 percent, furthermore, if the large number of undiagnosed cases is taken into account (31), it could fall another order of magnitude to a few tenths of a percent, which is similar to the seasonal flu. While having a new pandemic flu-like pneumonia that occasionally flare-up is not a future anyone wants to be in, and it should not be a cause of public panic.

Many of the current methods of prevention and control in China are similar to wartime measures, such as rapid construction of new hospitals, extensive restrictions on the movement of people, delaying the start of school, high levels of martial law in communities, banning outsiders, and quarantining the residents of a building if a patient is found. These measures are likely to be unsustainable, and will put an unbearable strain on socioeconomic activity in the long term. It may be necessary to start setting a timetable for the withdrawal of such war measures, depending on the situation of each city. Meanwhile, the Chinese government should start focusing on developments towards more universal, milder measures for the diagnosis and treatment of COVID-19, such as tiered diagnosis and treatments, and regular disinfection of public appliances. 
The media around the world is doing an excellent job in covering all aspects of COVID-19, and raising public awareness, however, this exerts enormous pressure on governments to make over-drastic policies (59). This media portrait of a "killer virus" can only harm efforts to implement a successful and safe infection control strategy (60). It may be time for the media and education to start adjusting their tone to reduce unnecessary panic. Instead, the emphasis should move towards the popularization of public health, such as wearing masks when sick, washing hands frequently, coughing and sneezing etiquette, etc.

After SARS died down, much of the research and drug development related to SARS stopped because of lack of funding and market prospects. A simple bibliometric analysis from PubMed databases, using "SARS" as a search term, reveals around 800 articles in 2003, which dropped to 27 in 2019. Given that the new coronavirus invades the human body in a similar way to SARS, if many of the previous studies had persisted, we might be better prepared scientifically and medically for the new coronavirus. Perhaps we should learn from this lesson and rethink whether our entire scientific system is paying enough attention to infectious diseases.

\section{Conclusion}

Through consolidating foreign citizen evacuation data, the prevalence rate in Wuhan population is found to be around one percent, or 110000 infected people.

The large number of patients and the strong occult nature are two big problems, making the virus difficult to eradicate. The virulence and transmissibility of the new virus approach that of the influenza, and it would not be entirely unacceptable to tolerate a degree of persistence. We need to contemplate the possibility of long-term co-existence with COVID-19, rather than allowing overly-drastic measures to affect our normal lives and the normal functioning of the country's social and economic activities.

The above discussion is based on current knowledge of the new coronavirus and the new coronavirus pneumonia, which may change, as may the virus and the disease itself. We need to be very cautious in the face of an enemy we have never seen before.

\section{Conflict of Interest Statement}

The authors declare no conflict of interest.

\section{Acknowledgement}

The authors acknowledge some insight contribution from Professor Liming Wang from Zhejiang University.

1. Cheng ZJ, Shan J. 2019 Novel Coronavirus: Where We are and What We Know. Infection. 2020; In Press. 
2. NHC. Outbreak notification. 2020;Available at

http://www.nhc.gov.cn/xcs/yqtb/list gzbd.shtml.

3. DXY. Epidemic map. 2020;Available at

https://3g.dxy.cn/newh5/view/pneumonia?from=timeline\&isappinstalled=0.

4. Su S, Wong G, Shi W, Liu J, Lai ACK, Zhou J, et al. Epidemiology, Genetic Recombination, and Pathogenesis of Coronaviruses. Trends Microbiol. 2016;24(6):490-502.

5. Zhong NS, Zheng BJ, Li YM, Poon, Xie ZH, Chan KH, et al. Epidemiology and cause of severe acute respiratory syndrome (SARS) in Guangdong, People's Republic of China, in February, 2003. Lancet. 2003;362(9393):1353-8.

6. Peiris JS, Guan Y, Yuen KY. Severe acute respiratory syndrome. Nat Med. 2004;10(12 Suppl):S88-97.

7. Zaki AM, van Boheemen S, Bestebroer TM, Osterhaus AD, Fouchier RA. Isolation of a novel coronavirus from a man with pneumonia in Saudi Arabia. N Engl J Med. 2012;367(19):1814-20.

8. Lee J, Chowell G, Jung E. A dynamic compartmental model for the Middle East respiratory syndrome outbreak in the Republic of Korea: A retrospective analysis on control interventions and superspreading events. J Theor Biol. 2016;408:118-26.

9. Lee JY, Kim YJ, Chung EH, Kim DW, Jeong I, Kim Y, et al. The clinical and virological features of the first imported case causing MERS-CoV outbreak in South Korea, 2015. BMC Infect Dis. 2017;17(1):498.

10. Cheng VCC, Wong SC, To KKW, Ho PL, Yuen KY. Preparedness and proactive infection control measures against the emerging Wuhan coronavirus pneumonia in China. J Hosp Infect. 2020.

11. Zhu N, Zhang D, Wang W, Li X, Yang B, Song J, et al. A Novel Coronavirus from Patients with Pneumonia in China, 2019. N Engl J Med. 2020.

12. Huang C, Wang Y, Li X, Ren L, Zhao J, Hu Y, et al. Clinical features of patients infected with 2019 novel coronavirus in Wuhan, China. Lancet. 2020; In Press.

13. WHO. Summary of probable SARS cases with onset of illness from 1 November 2002 to 31 July 2003. 2003.

14. WHO. MERS Monthly Report November 2019. 2019.

15. Biscayart C, Angeleri P, Lloveras S, Chaves TdSS, Schlagenhauf $P$, J.Rodríguez-Morales A. The next big threat to global health? 2019 novel coronavirus (2019-nCoV): What advice can we give to travellers? - Interim recommendations January 2020, from the Latin-American society for Travel Medicine (SLAMVI). Travel Medicine and Infectious Disease. 2020; In Press.

16. WHO. Novel Coronavirus (2019-nCoV) SITUATION REPORT. 2020.

17. Wu JT, Leung K, Leung GM. Nowcasting and forecasting the potential domestic and international spread of the 2019-nCoV outbreak originating in Wuhan, China: a modelling study. The Lancet. 2020;Online First.

18. WHO. Consensus document on the epidemiology of severe acute respiratory syndrome (SARS). 2003.

19. WHO. WHO MERS Global Summary and Assessment of Risk. 2018.

20. About the present situation of new type coronavirus infectious disease and correspondence of Ministry of Health, Labor and Welfare (February 7 version). Ministry of Health, Labor and Welfare. 2020;Available at https://www.mhlw.go.jp/stf/newpage 09396.html.

21. The second batch of wuhan evacuees all tested negative and one was confirmed infected in the first batch. KBS. 2020;Available at

http://world.kbs.co.kr/service/news view.htm?lang=c\&Seq Code=66621.

22. Routine new headquarters of the Central Accident Management Division of New Coronavirus Infection 2020-02-01. Ministry of Health and Welfare. 2020;Available at https://www.mohw.go.kr/eng/nw/nw0101vw.jsp?PAR MENU ID=1007\&MENU ID=100701\&page= 1\&CONT SEQ=352718. 
23. New Outbreaks of Corona Virus in Korea. Ministry of Health and Welfare. 2020;Available at http://www.mohw.go.kr/react/al/sal0301vw.jsp?PAR MENU ID=04\&MENU ID=0403\&page=3\&CO NT SEQ=352645.

24. Waldrop T, Yan H. The 1st group of Americans evacuated from Wuhan and quarantined over coronavirus just got released. CNN. 2020;Available at https://edition.cnn.com/2020/02/10/us/coronavirus-american-evacuees-release/index.html.

25. Coronavirus: German evacuation flight from China carried two infected people. DW. 2020;Available at https://www.dw.com/en/coronavirus-german-evacuation-flight-from-chinacarried-two-infected-people/a-52229955.

26. AFP. Coronavirus: countries evacuate citizens from China. New Straits Times. 2020;Available at https://www.nst.com.my/world/world/2020/01/561086/coronavirus-countries-evacuate-citizenschina.

27. Wei TT. Some Singaporeans with symptoms of virus staying behind in Wuhan even as 92 are evacuated. The Straits Times. 2020;Available at https://www.straitstimes.com/singapore/somesingaporeans-with-symptoms-of-virus-staying-behind-in-wuhan-even-as-92-are-evacuated.

28. Yong C. Coronavirus: 2 new cases in S'pore, including Certis officer who had served quarantine orders on 2 who tested positive. The Straits Times. 2020;Available at https://www.straitstimes.com/singapore/coronavirus-2-new-cases-in-singapore-including-certiscisco-staff-who-had-served.

29. Seckin B. Italy reports third confirmed case of coronavirus. AA. 2020;Available at https://www.aa.com.tr/en/europe/italy-reports-third-confirmed-case-of-coronavirus/1726934.

30. Network CN. Health committee responds to 5 million people leaving Wuhan: our common enemy is disease, not Wuhan. Sina News. 2020;Available at https://news.sina.com.cn/c/2020-0129/doc-iihnzhha5261128.shtml.

31. magazine C. People outside the statistics: do they die of "common pneumonia"? Sohu. 2020;Available at http://www.sohu.com/a/370032279 120094087.

32. WHO. Novel Coronavirus(2019-nCoV) Situation Report - 7. 2020.

33. Rothe C, Schunk M, Sothmann P, Bretzel G, Froeschl G, Wallrauch C, et al. Transmission of 2019-nCoV Infection from an Asymptomatic Contact in Germany. N Engl J Med. 2020.

34. Kupferschmidt K. Study claiming new coronavirus can be transmitted by people without symptoms was flawed. Science. 2020.

35. Xinhua. China's CDC detects a large number of new coronaviruses in the South China seafood market in Wuhan. 2020;Available at http://www.xinhuanet.com/2020-

01/27/c 1125504355.htm.

36. Cohen J. Wuhan seafood market may not be source of novel virus spreading globally. Science. 2020.

37. Li Q, Guan X, Wu P, Wang X, Zhou L, Tong Y, et al. Early Transmission Dynamics in Wuhan, China, of Novel Coronavirus-Infected Pneumonia. N Engl J Med. 2020;In Press.

38. Zhou P, Yang X-L, Wang X-G, Hu B, Zhang L, Zhang W, et al. Discovery of a novel coronavirus associated with the recent pneumonia outbreak in humans and its potential bat origin. bioRxiv. 2020.

39. Guo Q, Li M, Wang C, Wang P, Fang Z, Tan J, et al. Host and infectivity prediction of Wuhan 2019 novel coronavirus using deep learning algorithm. bioRxiv. 2020.

40. Kan $B$, Wang $M$, Jing $H, X u H$, Jiang $X$, Yan $M$, et al. Molecular evolution analysis and geographic investigation of severe acute respiratory syndrome coronavirus-like virus in palm civets at an animal market and on farms. J Virol. 2005;79(18):11892-900.

41. Sabir JS, Lam TT, Ahmed MM, Li L, Shen Y, Abo-Aba SE, et al. Co-circulation of three camel coronavirus species and recombination of MERS-CoVs in Saudi Arabia. Science. 2016;351(6268):81-4. 42. Ji W, Wang W, Zhao X, Zai J, Li X. Homologous recombination within the spike glycoprotein of the newly identified coronavirus may boost cross-species transmission from snake to human. J Med Virol. 2020. 
43. News. South China Agricultural University Press Release. 2020;Available at https://news.163.com/20/0207/11/F4PF1AN800018990.html.

44. Chan JF, Yuan S, Kok KH, To KK, Chu H, Yang J, et al. A familial cluster of pneumonia associated with the 2019 novel coronavirus indicating person-to-person transmission: a study of a family cluster. Lancet. 2020; In Press.

45. Iuliano AD, Roguski KM, Chang HH, Muscatello DJ, Palekar R, Tempia S, et al. Estimates of global seasonal influenza-associated respiratory mortality: a modelling study. Lancet. 2018;391(10127):1285-300.

46. Taubenberger JK, Morens DM. 1918 Influenza: the mother of all pandemics. Emerg Infect Dis. 2006;12(1):15-22.

47. Biggerstaff M, Cauchemez S, Reed C, Gambhir M, Finelli L. Estimates of the reproduction number for seasonal, pandemic, and zoonotic influenza: a systematic review of the literature. BMC Infect Dis. 2014;14:480.

48. CDC. Prevention and Control of Influenza Recommendations of the Advisory Committee on Immunization Practices (ACIP).

49. Litvinova M, Liu QH, Kulikov ES, Ajelli M. Reactive school closure weakens the network of social interactions and reduces the spread of influenza. Proc Natl Acad Sci U S A.

2019;116(27):13174-81.

50. Moghadas SM, Haworth-Brockman M, Isfeld-Kiely H, Kettner J. Improving public health policy through infection transmission modelling: Guidelines for creating a Community of Practice. Can J Infect Dis Med Microbiol. 2015;26(4):191-5.

51. Bootsma MC, Ferguson NM. The effect of public health measures on the 1918 influenza pandemic in U.S. cities. Proc Natl Acad Sci U S A. 2007;104(18):7588-93.

52. Gunaratnam PJ, Tobin S, Seale H, Marich A, McAnulty J. Airport arrivals screening during pandemic (H1N1) 2009 influenza in New South Wales, Australia. Med J Aust. 2014;200(5):290-2.

53. Nishiura H, Kamiya K. Fever screening during the influenza (H1N1-2009) pandemic at Narita International Airport, Japan. BMC Infect Dis. 2011;11:111.

54. CDC. CDC Seasonal Flu Vaccine Effectiveness Studies.

55. Plotkin S, Robinson JM, Cunningham $G$, Iqbal R, Larsen $S$. The complexity and cost of vaccine manufacturing - An overview. Vaccine. 2017;35(33):4064-71.

56. Pong W. A dozen vaccine programs under way as WHO declares coronavirus public health emergency. Biocentry. 2020.

57. Feng E, Cheng A. In Quarantined Wuhan, Hospital Beds For Coronavirus Patients Are Scarce. NPR. 2020.

58. PICKRELL R. Wuhan is scrambling to fill 11 sports centres, exhibition halls, and other local venues with over 10,000 beds to create makeshift coronavirus hospitals. Business Insider. 2020.

59. Vergano D. Don't Worry About The Coronavirus. Worry About The Flu. Buzzfeed News. 2020.

60. The L. Emerging understandings of 2019-nCoV. Lancet. 2020. 